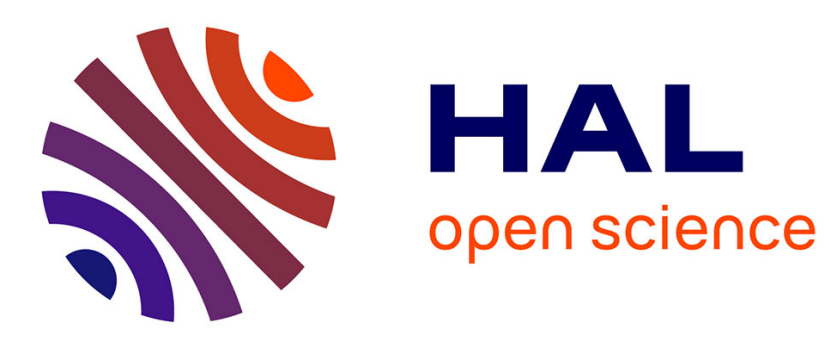

\title{
How to handle gender and number agreement in statistical language models?
}

\author{
Caroline Lavecchia, Kamel Smaïli, Jean-Paul Haton
}

\section{To cite this version:}

Caroline Lavecchia, Kamel Smaili, Jean-Paul Haton. How to handle gender and number agreement in statistical language models?. Ninth International Conference on Spoken Language Processing INTERSPEECH 2006, Sep 2006, Pittsburgh, Pennsylvania/USA. inria-00103497

\section{HAL Id: inria-00103497 https://hal.inria.fr/inria-00103497}

Submitted on 4 Oct 2006

HAL is a multi-disciplinary open access archive for the deposit and dissemination of scientific research documents, whether they are published or not. The documents may come from teaching and research institutions in France or abroad, or from public or private research centers.
L'archive ouverte pluridisciplinaire HAL, est destinée au dépôt et à la diffusion de documents scientifiques de niveau recherche, publiés ou non, émanant des établissements d'enseignement et de recherche français ou étrangers, des laboratoires publics ou privés. 


\title{
How to handle gender and number agreement in statistical language models?
}

\author{
Caroline Lavecchia, Kamel Smailli and Jean-Paul Haton
}

\author{
INRIA-LORIA, Speech Group \\ B.P. 239 - 54602 Villers les Nancy, France \\ Tel.: +33 (0)383592083 - Fax: +33(0)383278319 \\ e-mail: \{lavecchi, smaili,jph\}@ loria.fr - http://www.loria.fr/equipes/parole
}

\begin{abstract}
The agreement in gender and number is a critical problem in statistical language modeling. One of the main difficulties in speech recognition of French language is the presence of misrecognized words due to the bad agreement (in gender and number) between words. Statistical language models do not treat this phenomena directly. This paper focuses on how to handle the issue of this agreement. We introduce an original model called Features-Cache (FC) to estimate the gender and the number of the word to predict. It is a dynamic variable-length Features-Cache. The size of the cache is automatically determined in accordance to syntagm delimitors. The main advantage of this model is that there is no need to any syntactic parsing : it is used as any other statistical language model. Several models have been carried out and the best one achieves an improvement of approximatively 9 points in terms of perplexity. This model has been integrated in a speech recognition system based on JULIUS engine. Tests have been carried out on 280 sentences provided by AUPELF for the French automatic speech recognition evaluation campaign. This new model outperforms the baseline one, in terms of word error, by $3 \%$.
\end{abstract}

Index Terms : statistical language modeling, features, Features Cache, Partial Features Cache.

\section{Introduction}

In current statistical language models, it is difficult to take into account the agreement between two words, especially when they are not close. In such models, the agreement is hidden in the probabilities assigned to the sentence n-grams. In French, each word has several linguistic features, and some of them may be incompatible with the features of another word. For instance, the production of a word is affected by the gender and number of its left context. Statistical language models handle the gender and number agreement inadequately, and that contributes to reduce the performance of the French speech recognition systems. For instance, the sentence Les pommes que j'ai mangées étaient vertes ${ }^{1}$ is often recognized as Les pommes que j'ai mangé était verte ${ }^{2}$. French is an inflected language with several homonyms words, consequently linguistic features are very useful to reduce speech recognition errors due to this phenomena. Few research works have been conducted in this area [1] [2], and we consider that the introduction of such information will improve the statistical language models. Long distance dependencies in statistical language modeling have been widely

\footnotetext{
${ }^{1}$ The apples I ate were green

${ }^{2}$ The words mangées and mangé are acoustically identical
}

explored in the litterature even by using syntactic structure [3] Our work is related to long distance features dependencies without introducing any parsing nor syntactic rules.

The model we propose, Features-Cache (FC), is inspired from the classical cache model [4]. Henceforth, in our model a word depends not only on its word left context, but also on its gender and number left contexts. The idea is to capture the left context features in order to predict if a word is compatible in terms of features.

\section{The Features-Cache model}

\subsection{The Cache Model}

The Cache model supposes that a word which occured in the recent past is much more likely to be used sooner than indicated by its frequency in the language. That leads to conclude that a classical n-gram is less powerful than a Cache to predict the recent uttered words. The Cache model estimates the probability of a word from its recent frequency of use.

$$
P\left(w_{i}\right)=\frac{1}{N} \sum_{j=1}^{N} \delta\left(w_{i}, w_{j}\right)
$$

where $N$ is the length of the cache and $\delta\left(w_{i}, w_{j}\right)$ is the Kronecker function which is equal to 1 if $w_{i}=w_{j}$ and 0 otherwise.

\subsection{An outline of the Features-Cache}

Since a Cache is more efficient to predict a word which occurred in the recent past, we have extended this idea to word features. We propose a new model which takes into account the recent word features in order to predict a compatible word in terms of features. For instance, in the sentence : Les pommes sont vertes ${ }^{3}$, the feature "number" of vertes is compatible with its past, the gender of this word is also compatible with Les and pommes. In French, some words are insensitive to gender or number, that means that some words may have the same orthographic form in singular and plural as corps, souris, etc. Other words are invariant in gender as égoïste, tranquille, etc. Consequently, the mass of words having one of both the features could be unbalanced. For this reason, we split the Features-Cache model into two Features-Cache models : the gender Features-Cache and the number Features-Cache. Under this assumption, we propose to estimate the Features-Cache probability of a word as follows :

\footnotetext{
${ }^{3}$ The apples are green
} 


$$
\begin{aligned}
P_{F C}\left(w_{i}\right)= & \delta \frac{N\left(G\left(w_{i}\right)\right)}{\sum_{w_{j} \in V} N\left(G\left(w_{j}\right)\right)} \\
& +\lambda \frac{N\left(U\left(w_{i}\right)\right)}{\sum_{w_{j} \in V} N\left(U\left(w_{j}\right)\right)}
\end{aligned}
$$

where $N(f(x))$ is the occurrence of the feature $f$ of a word $x$ occured in Cache, $G$ the gender feature and $U$ the number feature and $V$ is the vocabulary. Table 1 presents features we used in our model.

\begin{tabular}{|c|c|}
\hline Feature & Example \\
\hline FS (Female - Singular) & porte \\
\hline MS (Male - Singular) & stylo \\
\hline FP (Female - Plural) & portes \\
\hline MP (Male - Plural) & stylos \\
\hline Fi (Female - Invariant in number) & souris \\
\hline Mi (Male - Invariant in number) & tapis \\
\hline iS (Invariant in gender - Singular) & égoïste \\
\hline iP (Invariant in gender - Plural) & ces \\
\hline ii (Invariant in gender and number) & beaucoup \\
\hline
\end{tabular}

TAB. 1 - Features list used in the Features-Cache model

\begin{tabular}{|c|c|c|c|c|}
\hline & Mean & Min & Max & $\sigma$ \\
\hline$\alpha$ & 0.933 & $10^{-6}$ & 1 & 0.151 \\
\hline$\beta$ & 0.064 & $10^{-6}$ & 1 & 0.151 \\
\hline
\end{tabular}

TАВ. 2 - Statitics on EM parameters interpolation

Obviously, this model cannot be used alone ; it is linearly interpolated with a classical $\mathrm{n}$-gram. The estimation of a word $w_{i}$ given a left context $h$ is calculated as follows :

$$
P\left(w_{i} \mid h\right)=\alpha P_{n g r a m}\left(w_{i} \mid h\right)+\beta P_{F C}\left(f\left(w_{i}\right) \mid \text { Cache }\right)
$$

where Cache is a sequence of $m$ features, and $\alpha, \beta$ are the interpolation parameters. In table 2 some statistics on the interpolation parameters (mean, min, max and standard deviation) are presented. Figure 1 illustrates the evolution of $\alpha$ and $\beta$ obtained by EM algorithm.

\section{Data description}

Experiments were performed on Le Monde newspaper corpus. The training corpus contains 32 million words, the development 8 million words and the test 1,8 million words. The vocabulary is made up of the 57000 most frequent words. The features of words are extracted from a French lexical database (BDLEX distributed by ELRA) ${ }^{4}$ which contains 430000 words. This database contains the inflected words derived from the canonical words. Each entry includes spelling, pronunciation, morphosyntactic attributes and a frequency indicator. The length of the Cache-Features has been set experimentally to 5 . This is due to the fact that the agreement in gender and number has to be done in a close context.

\footnotetext{
${ }^{4}$ Base de Données LEXicales
}

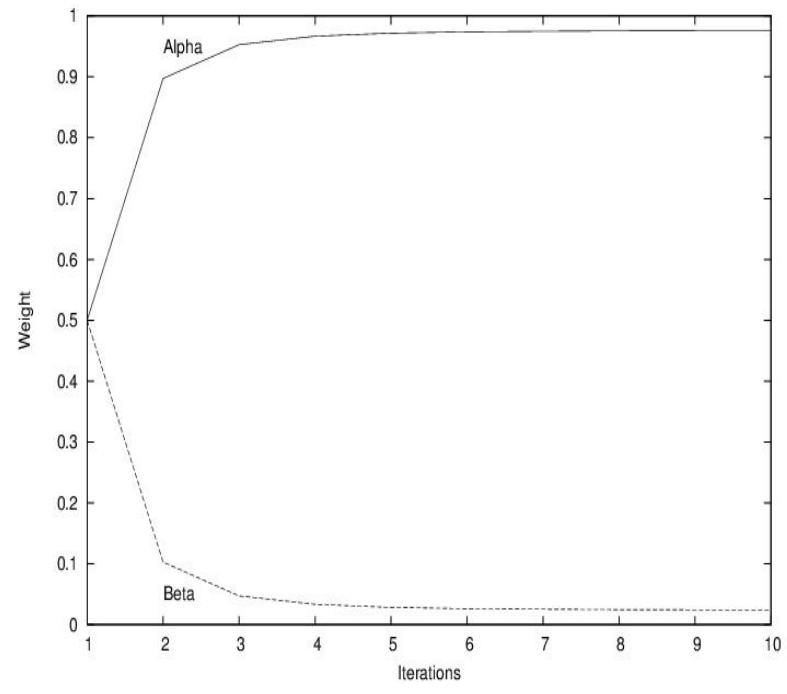

FIG. 1 - Evolution of interpolation parameters

\section{Results and discussion}

In order to perform the Features-Cache language model, we labeled each word with its features by using BDLEX database. In the following, interpolation parameters have been optimized with EM algorithm [5]. Two sets of parameters have been calculated : one parameter by model in the mixture (1byM) and one parameter by history $(1 \mathrm{byH})$ for interpolated bigrams as shown in table 3. Experiments show that the model is promising and the use of

\begin{tabular}{|l|c|c|c|}
\hline & Baseline & \multicolumn{2}{|c|}{ FCache model } \\
\hline n-gram size & n-gram & 1byM & 1byH \\
\hline$P P L_{n=2}$ & 212.83 & 206.85 & 204.75 \\
$P P L_{n=3}$ & 165.35 & 159.42 & - \\
\hline
\end{tabular}

TАВ. 3 - Results on interpolated Features-Cache model

features bring an improvement of 8 points for an interpolated bigram and almost 6 points for an interpolated trigram. Several experiments have been achieved on different corpora and each time a reduction on test-perplexity has been observed. That shows the potential of our approach in terms of test-perplexity reduction, and we have succeed to introduce linguistic features in statistical language models. This is done without introducing any linguistic rule nor parsing technical. We have to mention that the use of a classical word Cache outperfoms the results we obtained with the combined FC model (by 3 points).

Our objective is to conduct several experiments and to develop other models in order to improve more again the perplexity and hope to outperform our own speech recognition system [6].In the next section we show how to go further by introducing a dynamic feature cache that we call Partial Features-Cache. 


\section{Feature-Class model}

In this section we define a Feature-Class model as a n-gram of feature classes, the probability of word $w_{i}$ is then defined as :

$$
P\left(w_{i} \mid h_{w_{i}}\right)=P\left(w_{i} \mid C_{w_{i}}\right) P\left(C_{w_{i}} \mid h_{C_{w_{i}}}\right)
$$

where $C_{x}$ denotes the feature class of $x, h_{w_{i}}$ is the history of $w_{i}, h_{C_{w_{i}}}$ is the class history of $C_{w_{i}}$, and $P\left(w_{i} \mid C_{w_{i}}\right)$ computes the ratio of the word $w_{i}$ to the number of words having the same features as $w_{i}$ :

$$
P\left(w_{i} \mid C_{w_{i}}\right)=\frac{N\left(w_{i}\right)}{N\left(C_{w_{i}}\right)}
$$

where $N(x)$ is the occurrence of $x$ in the training corpus. We used 9 feature classes described previously in table 1 . The conditional probability $P\left(C_{w_{i}} \mid h_{C_{w_{i}}}\right)$ is estimated as a classical n-gram, where grams are replaced by their corresponding features.

\begin{tabular}{|l|c|c|c|}
\hline & Baseline & \multicolumn{2}{|c|}{ FClass model } \\
\hline n-gram size & n-gram & 1byM & 1byH \\
\hline$P P L_{n=2}$ & 212.83 & 211.82 & 209.78 \\
$P P L_{n=3}$ & 165.35 & 163.39 & - \\
\hline
\end{tabular}

TAв. 4 - Perplexities for baseline and Feature-Class

Unfortunately the class feature model leads to a weak improvement. Thus we decided to drop this model.

\section{Partial Features-Cache}

Experimental results show the interest of the Features-Cache, but this model may introduce errors in the relationship between word features. Actually, in the sentence Le portefeuille bleu de ma grande soeur est beau ${ }^{5}$, the word soeur, which is singular female, will be affected by the dominant features in the Cache (singular male) and consequetly its probability will decrease. In fact, because no parsing technique is conducted, we should only take into account the agreement between words inclosed on buckets or syntagms. In the previous example, the compatibility of features has to be checked inside the syntagms le portefeuille bleu or ma grande soeur, the word beau has to be treated with a distant model [7], [8]. Actually, the features of the word soeur have to be checked inside the last syntagm. With this assumption, the cache has to be splitted into buckets (or syntagms). The decomposition of a word history leads to what we call a Partial Features-Cache. For that, we have to find out the limits of linguistic groups or syntagms. To deal with this issue, we use a list of tool words as prepositions, conjunctions, etc, which are unvarying in gender and number, in order to set the limits of groups. These separators permit to retrieve dynamically the adequate size of the features-Cache, leading to what we call a partial Features-Cache. In the previous example, the word de separates two syntagms. The results presented in table 5 were obtained by using the separators $d e$ and $d u$. Despite the slight improvement, we are convinced that the agreement in gender and number has to be considered inside a group of words delimited by separators. In order to go further, we introduce other separators (table 6). The introduction of these separators leads to an interpolated bigram perplexity of 203.95 (table 7) and an interpolated trigram perplexity of 159.18 . Overall, our approach allows to decrease the

\footnotetext{
${ }^{5}$ The blue wallet of my older sister is beautiful
}

\begin{tabular}{|l|c|c|c|}
\hline & Baseline & \multicolumn{2}{|c|}{ Partial Features-Cache } \\
\hline n-gram size & n-gram & 1 byM & 1byH \\
\hline$P P L_{n=2}$ & 212.83 & 206.57 & 204.43 \\
$P P L_{n=3}$ & 165.35 & 159.19 & - \\
\hline
\end{tabular}

TAв. 5 - Results on Partial Features-Cache using separators de and $d u$

\begin{tabular}{|c|c|c|c|c|c|c|}
\hline de & du & mais & ou & et & donc & or \\
\hline ni & car & dans & avant & depuis & que & qui \\
\hline
\end{tabular}

TAB. 6 - Syntagm word separators

bigram perplexity by almost 9 points. We have to continue our investigation in order to delimit more correctly the boundaries of syntagms in a Features-Cache. We decided to analyse the weights

\begin{tabular}{|l|c|c|c|}
\hline & Baseline & \multicolumn{2}{|c|}{ Partial Features-Cache } \\
\hline n-gram size & n-gram & 1 byM & 1byH \\
\hline$P P L_{n=2}$ & 212.83 & 206.56 & 203.95 \\
$P P L_{n=3}$ & 165.35 & 159.18 & - \\
\hline
\end{tabular}

TAв. 7 - Perplexities on Partial Features-Cache using an extended list of sepators

assigned to the Features-Cache model, this study shows that only 3655 histories have a weight greater than 0.3 and only 736 among them have weights which exeed 0.6. Table 8 gives some histories and the corresponding Feature-Cache ponderation which are significant. Despite the weak number of histories, the contribution of Partial Features-Cache brings an improvement over a n-gram.

\begin{tabular}{|c|c||c|c|}
\hline Word & FC weight & Word & FC weight \\
\hline Derrick & 0 & apportée & 1 \\
\hline Tiozzo & 0.06 & conceptuels & 1 \\
\hline increvable & 0.19 & concertation & 0.99 \\
\hline fuseaux & 0.21 & concurrente & 0.99 \\
\hline défaits & 0.26 & voies & 0.90 \\
\hline Sun & 0.27 & restaient & 0.78 \\
\hline votre & 0.31 & ressentons & 0.76 \\
\hline arrière-goût & 0.36 & verdeur & 0.61 \\
\hline
\end{tabular}

TAB. 8 - Some histories leading to significant weights (left part)

\section{Speech recognition results}

\subsection{An overview of the speech engine}

In order to evaluate our approach in a speech recognition system, we used the JULIUS speech engine. JULIUS is an open source engine recognition originally developed by Akinobu Lee at Kyoto university [9]. Two passes are performed. In the first pass a tree-structured lexicon associated to a bigram is applied with the frame-synchronous beam search algorithm. This first pass produces a word lattice. The second pass is based on a trigram 
model and researches the best sentence in the word lattice.

\subsection{Implementation}

In the first pass we use a standard bigram trained on ten years extracted from the French newspaper Le Monde. The acoustic model is based on HMM phone without adaptation to gender or speaker. In the second pass, we integrate our Partial Features-Cache presented in the previous section. Tests have been carried out on 280 sentences provided by AUPELF for the French automatic speech recognition evaluation program [10].

\subsection{Analysis and Review}

Table 9 shows the results obtained by integrating a Partial Features-Cache in the second pass instead of the standard trigram. This new language model has a real impact and achieves an improvement of $3 \%$ of the word error. We can also notice that all the bold rates of table 9 indicate slight improvements in comparison to the corresponding rates of the standard recognition engine.

\begin{tabular}{|c||c|c||c|c|c|c|c|}
\hline Model & \#Snt & \#Wrd & Corr & Sub & Del & Ins & Err \\
\hline \hline Baseline & 134 & 4076 & $\mathbf{6 1 . 8}$ & $\mathbf{2 5 . 2}$ & 13.1 & $\mathbf{1 . 9}$ & $\mathbf{4 0 . 2}$ \\
\hline P-F-C & 134 & 4076 & $\mathbf{6 2 . 4}$ & $\mathbf{2 4 . 0}$ & 13.6 & $\mathbf{1 . 4}$ & $\mathbf{3 9 . 0}$ \\
\hline
\end{tabular}

TAB. 9 - Performance in terms of word error

\section{Conclusion}

In this paper, we presented an original statistical language model based on features of words. The idea is to consider a word not only as an orthographic form, but as a linguistic unit with several attributes. In this work, we focused on only two features : gender and number. Several feature models, based on statistical language formalisms have been developed, in order to find the best one. The features have been considered inside a left short window of the word to predict. Significant performance have been achieved with a variable-length Cache (Partial Features-Cache). The interpolated bigram test-perplexity has been decreased by almost 9 points. For the interpolated trigram, despite using a sub-optimal weights for both combined models, an improvement of more than 6 points has been obtained. With these results, we showed the feasibility of the features language model concept and the easiness way to formalize them. The Partial Features-Cache model has also been integrated in the second decoding pass of the speech recognition system. An improvement of 1.2 points of the WER has been reached.

In future works, we will conduct experiments in order to take into account other agreements, such as the agreement between a subject and its verb. In this scope, new features will be introduced. Thereafter, we will be interested in automatically new agreements according to features considered.

\section{Acknowledgements}

This research is supported by EADS foundation in the framework of speech-to-speech translation Ph.D thesis. This work begun before the official start day of Ph.D.

The authors whish to thank Dr. David Langlois and Dr. Armelle Brun for their precious help.

\section{References}

[1] J. A. Bilmes and K. Kirchhoff, "Factored language models and generalized parallel backoff," in Proceeding of $\mathrm{Hu}$ man Language Technology Conference, Edmonton, Canada, 2003.

[2] K. Smali, S. Jamoussi, D. Langlois, and J. P. Haton, "Statistical feature language model," in Proc. ICSLP, Jeju, 2004.

[3] C. Chelba and F. Jelinek, "Exploiting syntactic structure for language modeling," in Proceedings of the Thirty-Sixth Annual Meeting of the Association for Computational Linguistics and Seventeenth International Conference on Computational Linguistics, 1998, pp. 225-231.

[4] R. Kuhn and R. DeMori, "A cache-based natural language model for speech recognition," IEEE Trans. PAMI, vol. 12, no. 6, pp. 570-582, 1990.

[5] A. Dempster, N. Laird, and D. Rubin, "Maximum likelihood from incomplete data via the em algorithm," Journal of the Royal Statistical Society B, vol. 39, pp. 1-38, 1977.

[6] Armelle Brun, Christophe Cerisara, Dominique Fohr, Irina Illina, David Langlois, and Odile Mella, "Ants le système de transcription automatique du loria," in Workshop ESTER, Avignon, France, Mar 2005.

[7] X. Huang and al., "The sphinx speech recognition system : an overview," Computer speech and Language, vol. 2, pp. 137-148, 1993.

[8] D. Langlois and K. Smaili, "A new based distance language model for a dictation machine : application to maud," in Proc. EUROSPEECH, 1999, pp. 1779-1782.

[9] A. Lee, T. Kawahara, and K. Shikano, "Julius - an open source real-time large vocabulary recognition engine," in Proc. EUROSPEECH, 2001, pp. 1691-1694.

[10] G. Adda, M. DeCalmes, L. Lamel, G. Perennou, M. Rajman, S. Rosset, and J. Zeiliger, "Ressources pour l'apprentissage, le dveloppement et l'valuation des systmes de dicte vocale en français : corpus de texte, de parole et lexical," in Actes des premires JST Francil 1997, Avignon, France, April 1997, pp. 305-309. 